ISSN 1997-5902

\title{
Les plantes à alcaloïdes utilisées par les populations de la ville de Douala (Cameroun)
}

\author{
Yinyang J1, Mpondo Mpondo E¹, Tchatat M², Ndjib RC ${ }^{3}$, Mvogo Ottou PB ${ }^{3}$, Dibong SD(1,3,4) \\ ${ }^{1}$ Département des Sciences Pharmaceutiques, Faculté de Médecine et des Sciences \\ Pharmaceutiques, Université de Douala, B.P. 2701 Douala, Cameroun \\ 2 Institut de Recherche Agricole pour le Développement (IRAD), B.P. 2123 Yaoundé, Cameroun \\ 3 Département de Biologie des Organismes Végétaux, Faculté des Sciences, B.P. 812 Douala, \\ Cameroun \\ ${ }^{4}$ Département d'Aquaculture, Institut des Sciences Halieutiques, B.P. 2701 Douala, Cameroun \\ Auteur correspondant : didierdibong@yahoo.fr
}

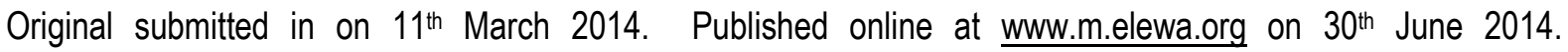
http://dx.doi.org/10.4314/jab.v78i1.7

\section{RESUME}

Objectif : Une enquête ethnobotanique a été conduite de janvier à février 2013 dans la ville de Douala concernant les différents usages des plantes à alcaloïdes par les populations.

Méthodologie et résultats : Une dizaine de quartiers ont été prospectés pour le recensement des plantes à alcaloïdes. Ces prospections ont permis de conduire des enquêtes semi-structurées basées sur les interrogations directes, concernant les usages thérapeutiques de ces plantes par les populations ciblées. Au total, 73 espèces végétales à alcaloïdes dont 21 herbacées et 52 ligneuses ont été inventoriées. Parmi ces espèces, 30 sont locales et 43 exotiques. Elles sont reparties en 62 genres et 40 familles. Le ravitaillement se fait dans les champs, les forêts secondarisées, les jardins de cases et vergers, les jachères et marchés. Les espèces de la famille des Solanaceae, Apocynaceae et Rubiaceae sont pour la plupart riche en divers alcaloïdes. Les feuilles, les écorces et les racines sont les parties les plus utilisées dans les recettes pour le traitement de diverses maladies. La voie orale étant le mode d'administration par excellence des dites recettes. Les métabolites secondaires, dont les alcaloïdes, sont inégalement répartis dans les plantes. Ils sont produits dans un organe, tissu ou type cellulaire spécifique à des stades particuliers du développement de la fleur, du fruit, de la graine ou de la plantule. Les plantes à alcaloïdes sont utilisées comme antalgiques majeurs, antipaludéens et pour combattre l'excès d'acide urique. Elles sont également utilisées comme stupéfiants, laxatifs, aphrodisiaques et comme cholinergiques ou comme anticancéreux. Elles entrainent l'accélération du rythme cardiaque et stimulent le système nerveux central avec une augmentation de la vigilance.

Conclusion et application des résultats : Toutes ces propriétés peuvent justifier l'intégration de ces produits forestiers non ligneux dans les programmes d'aménagement forestier, en vue de leur exploitation soutenue et durable.

Mots-clés : Solanaceae, Apocynaceae, Rubiaceae, alcaloïdes, exploitation

\section{ABSTRACT}

Objective: An ethnobotanical survey was conducted from January to February 2013 in Douala town relating to the various uses of alkaloids plants species by the people.

Methodology and results: Ten districts were surveyed for the census alkaloid plants. These surveys helped conduct semi-structured surveys based on direct questions concerning the therapeutic uses of these plants by the targeted populations. A total of 73 alkaloid plant species were identified of which 21 were 
herbaceous and 52 woody. Among these species, 30 are indigenous and 43 exotic. They were distributed in 62 genera belonging to 40 families. The supply is provided from farms, secondary forests, gardens, fallows and markets. Solanaceae, Apocynaceae and Rubiaceae are rich in various alkaloids. Leaves, barks and roots are the plant parts mostly used in recipes for the treatment of various diseases. Drugs are mainly administered through the oral root. Secondary metabolites, including alkaloids are unevenly distributed in plants. They are produced at particular stages of development of the flower, fruit, seed or seedling in a specific organ, tissue or cell. Alkaloid plant species are used as major analgesics, antimalarial and to fight against excess uric acid. They are also used as drugs, laxatives, aphrodisiac and as cholinergic or as anticancer agents. They increased heart rate and stimulate the central nervous system with increased vigilance.

Conclusion and application of results: All these properties can justify the inclusion of these non timber forest products in forest management programs for their sustainable exploitation.

Keywords : Solanaceae, Apocynaceae, Rubiaceae, alkaloids, exploitation

\section{INTRODUCTION}

Les plantes, éléments vitaux de la diversité biologique servent essentiellement au bien être humain (Adjanohoun et al., 1996, 2000). En dehors des plantes cultivées, plusieurs plantes sauvages peu connues revêtent une grande importance culturelle et un fort potentiel économique pour l'alimentation, les soins, l'énergie, l'habillement et la construction des logements. Les relations entre les plantes et les hommes existent depuis de longues dates (Baytop, 1999, Din et al., 2011). Les médecines « douces ", particulièrement la phytothérapie, connaissent un succès considérable dans nombreuses régions d'Afrique, d'Asie et d'Europe. Des enquêtes récentes $80 \%$ des populations rurales des pays en développement et $85 \%$ des populations au sud du Sahara utilisent les plantes médicinales comme principal traitement et la majeure partie des thérapies implique l'exploitation du principe actif des plantes médicinales (OMS, 2002 ; Biyiti et al., 2004 ; Dibong et al., 2011a, c ; Mpondo et Dibong, 2012). De plus, ces plantes constituent des ressources inestimables pour l'industrie pharmaceutique (Awono et al., 2009). Après les progrès fulgurants de la botanique systématique (Linné, Jussieu et beaucoup d'autres) vint l'heure de la première édition de la Pharmacopée française (1818) et le règne des chimistes qui isolèrent une série impressionnante de molécules : morphine (1817), codéine (1832), acide salicylique et, dans la seconde moitié du XIX siècle : quinine, strychnine, colchicine, cocaïne, ésérine (Mpondo et al., 2012). En Afrique, le pouvoir thérapeutique des plantes était connu par les ancêtres et les parents de façon empirique (Nacoulma, 1996). Pour parvenir à une amélioration de cette médecine africaine, plusieurs investigations phytochimiques ont été faites afin d'apporter une justification scientifique quant à I'utilisation traditionnelle des plantes médicinales (N'Guessan et al., 2009). De nombreux produits ne participant pas au métabolisme de base de la plante ont été isolés et leurs effets thérapeutiques démontrés (Akroum, 2011). Parmi ces composés, les alcaloïdes représentent un groupe important de part leurs nombreux avantages biologiques, notamment thérapeutiques, pharmaceutiques et alimentaires. Les alcaloïdes sont des composés hétérocycliques azotées et basiques, qui dérivent des acides aminés. De nombreux travaux ont d'ailleurs montré l'intérêt des alcaloïdes dans l'activité antiplasmodiale, antispasmodique, anticancérale. Leurs effets laxatif et antirhumatismal, antalgique et analgésique ont aussi été révélés (Zirihi et al. 2005; 2006; 2007; N'Guessan et al., 2009). Toutefois, dans la pharmacopée traditionnelle camerounaise, les connaissances sur les plantes à alcaloïdes restent embryonnaires et éparses. Le présent travail a pour but de pallier à ces lacunes. Les objectifs spécifiques envisagés sont: (1) de recenser toutes les plantes à alcaloïdes connues par les populations de la ville de Douala et (2) d'inventorier leurs usages ethnobotaniques.

\section{MATERIEL ET METHODES}


Site d'étude : Douala (latitude, $03^{\circ} 40-04^{\circ} 11^{\prime} \mathrm{N}$; longitude, $09^{\circ} 16^{\prime}-09^{\circ} 52^{\prime} \mathrm{E}$; altitude, $13 \mathrm{~m}$ ) a un climat qui appartient au domaine équatorial d'un type particulier dit « camerounien » qui se caractérise par deux saisons avec une longue saison de pluies (au moins 9 mois), des précipitations abondantes (environ $4000 \mathrm{~mm}$ par an), des températures élevées $\left(26,7^{\circ} \mathrm{C}\right)$ et stables. La moyenne minimale de température à Douala pour 30 années (1961-1990) est de $22,6^{\circ} \mathrm{C}$ en juillet et la moyenne maximale de température de 32,3 ${ }^{\circ} \mathrm{C}$ en février. L'humidité relative de l'air reste élevée toute l'année et voisine de $100 \%$ (Din et al., 2008). Douala, chef-lieu de la région du Littoral compte quatre départements : le Moungo, la Sanaga Maritime, le Nkam et le Wouri. Douala a environ 2,5 millions d'habitants et la croissance démographique n'est pas toujours suivie d'une augmentation des ressources économiques (Priso et al., 2011). La région du Littoral est un ensemble d'écosystèmes où se côtoient les forêts denses humides sempervirentes notamment la forêt dense humide sempervirente littorale ( 0 et $100 \mathrm{~m}$ d'altitude) et la forêt dense humide biafréenne (100 et $500 \mathrm{~m}$ d'altitude), la mangrove, les marécages, la végétation rupicole, les agro-écosystèmes et la végétation plus ou moins anthropisée (Letouzey, 1985). Enquêtes ethnobotaniques: L'identification des plantes à alcaloïdes a été faite à partir de la documentation botanique et ethnopharmacologique disponible dans les sites internet spécialisés: www.vulgaris-medical ;

www.universalis.fr/encyclopedie/alcaloïdes/; www.wow.com/plantes. Cette recherche a été confirmée sur le terrain par la localisation de chaque espèce dans son site écologique. Des enquêtes ethnobotaniques basées sur les interrogations directes portant sur les usages des plantes citées dans la pharmacopée traditionnelle et la pharmacopée populaire ont été conduites durant les mois de janvier et février 2013 dans les quartiers de la ville de Douala. L'approche des populations dont l'âge varie entre 35 et 50 ans a été basée sur le dialogue en langues française et locales. Cent vingt (120) personnes ont été enquêtées soient 30 personnes par quartier. Ces dernières devaient donner toutes les informations relatives aux plantes à alcaloïdes. Pour chaque personne qui acceptait de répondre aux questions et pour une maladie donnée, les informations ont été collectées selon un canevas standardisé inspiré des

\section{RESULTATS}

Au total, 73 espèces végétales à alcaloïdes reparties en 62 genres et 40 familles dont 21 herbacées et 52 ligneuses ont été inventoriées auprès des populations de la ville de Douala (Tableau 1). Les herbacées sont: Ageratum conyzoides Linn., Allium cepa Linn., Allium fiches Pharmel (Adjanohoun et al., 1989, 1994). Pour chaque indication thérapeutique, les détails de la recette et ont été relevés et la plante traitante récoltée. Les descriptions botaniques et les différents usages des plantes médicinales rencontrées ont été complétés par des informations fournies par des auteurs tels que Letouzey (1970), Béllé (1992), Thirakul (1995). Les échantillons ont été récoltés et conservés selon les techniques et méthodes de Schnell (1960). Les étudiants du niveau I du Département de Sciences Pharmaceutiques de la Faculté de Médecine et des Sciences Pharmaceutiques de l'Université de Douala ont été mis à contribution pour ce travail dans le cadre des travaux pratiques et du travail personnel de l'étudiant. Ils ont été répartis en 04 groupes de 20 étudiants soient 30 personnes enquêtées par groupe. Dans chaque groupe, la présence d'au moins un étudiant appartenant à la tribu majoritaire du quartier choisi a permis de communiquer et de réaliser sans difficulté majeure les enquêtes auprès des populations sollicitées. Les informations sur le « diagnostic des maladies » (symptômes ou effets physiologiques) ont été recueillies auprès des médecins, infirmiers ou agents de santé locaux et complétées par la revue bibliographique (Adjanohoun et al., 1989, 1996, 2000). Pour une exploitation pratique des données et une harmonisation avec le système international, les problèmes de santé cités ont été distingués en grands groupes de maladies selon la classification des maladies proposée par l'Organisation Mondiale de la Santé et adaptée par l'Organisation de l'Unité Africaine (OUA), pour la pharmacopée camerounaise et suivant la classification statistique internationale des maladies et des problèmes de santé connexes version 2010 (Adjanohoun et al., 1996 ; OMS, 1996, 2002 ; WHO, 2010). Des fiches d'enquête ont été élaborées dans le but de recueillir notamment des informations sur les parties de la plante utilisées, les modes de préparation, les maladies soignées, le mode d'administration et la posologie.

Collecte des données : Les échantillons des plantes ont été récoltés et leur identification validée par les botanistes de la Faculté des Sciences de l'Université de Douala et de l'herbier du jardin botanique de Limbé. Toutes les espèces nommées ont été répertoriées et identifiées. Les données de terrain ont été enregistrées sur un tableur Excel puis analysés.

sativum Linn., Apium graveolens Linn., Aloe vera Linn., Amaranthus hybridus L., Ananas comosus (L.) Merr., Brassica oleracea Linn., Cannabis sativa L., Cymbopogon citratus Stapf, Dissotis rotundifolia (Sm.) Triana., Emilia coccinea (Sims) G. Don, Ocimum 
basilicum L., Panax ginseng C.A. Mey., Panax quinquerfolium Herbs \& Health., Petroselinum sativum (Mill.) Nyman ex A. W. Hill., Solanum lycopersicum Mill., Solanum nigrum Linn., Solanum tuberosum Linn., Zea mays Linn., Zingiber officinale Rosc. Les ligneuses sont: Acanthus montanus T. Anderson, Adansonia digitata Linn., Alstonia boonei De Wild., Annona muricata Linn., Areca catechu L., Argemone mexicana L., Baillonella toxisperma Pierre., Borreria verticillata (L.) G.F.W. Meyer., Camellia sinensis (L.) Kuntze, Capsicum annuum L., Capsicum frutescens Linn., Carica papaya Linn., Catharanthus roseus (L.) G. Don., Chromolaena odorata (L.) King \& H.E. Robins., Cinchona calisaya Weed., Cinchona officinalis L., Cinchona pubescens Vahl., Citrus aurantifolia (Christm.) Swingle., Coffea arabica L., Coffea robusta Pierre ex A. Froehner., Cola acuminata (P. Beauv.) Schott \& Endl., Cola nitida Schott \& Endl, Combretum micranthum G. Don, Costus spicatus (Jacq.) Sw., Enantia chlorantha (Oliv.), Entandophragma cylindricum (Sprague)., Eremomastax speciosa (Hochst.) Cufod., Erythrophleum ivorense A. Chev., Eucalyptus globules Labill., Euphorbia hirta L., Garcinia kola Heckel., Hibiscus esculentus L., Hibiscus sabdarifa L., Holarrhena floribunda (G. Don.) Dur. \& Schinz, Kalanchoe crenata (Andrews) Haw., Mangifera indica Linn., Melissa officinalis L., Myristica fragrans Houtt., Nicotiana tabaccum L., Passiflora incarnata L., Pausinystalia yohimbe (K.Schum.) Pierre ex Beille., Persea Americana Mill., Phyllathus amarus Schumach. \& Thonn., Kongel. Danske Vidensk. Selsk. Skr., Psidium guajava Linn., Rauvolfia vomitoria Afzel., Senna alata Linn. (Robx.), Solanum melongena Linn., Theobroma cacao Linn., Thymus vulgaris Linn., Voacanga Africana Stapf, Voacanga thouarsii Roem. \& Schult.

Parmi les 73 espèces végétales à alcaloïdes recensées dans la ville de Douala, 30 sont endémiques et 43 sont

\section{DISCUSSION}

Les métabolites secondaires, dont les alcaloïdes sont inégalement répartis parmi les plantes, et varient qualitativement et quantitativement au sein des organes végétatifs d'une même plante. lls sont produits dans un organe, tissu ou type cellulaire spécifique à des stades particuliers du développement de la fleur, du fruit, de la graine ou de la plantule. Ils sont souvent synthétisés dans une partie de la plante et stockés dans une autre (Mpondo et al., 2012). Les feuilles, les écorces et les racines sont le siège par excellence de la biosynthèse et même du stockage des métabolites secondaires responsables des propriétés biologiques de la plante (Bitsindou, 1997). Aniszewski (2007) distingue les alcaloïdes vrais dérivant d'acides aminés et comportant un atome d'azote dans un système exotiques. Les espèces endémiques sont : Allium cepa, Adansonia digitata, Alstonia boonei, Annona muricata, Baillonella toxisperma, Capsicum annuum, Capsicum frutescens, Cinchona calisaya, Cinchona officinalis, Cinchona pubescens, Cola acuminata, Cola nitida, Costus spicatus, Emilia coccinea, Enantia chlorantha, Entandophragma cylindricum, Eremomastax speciosa, Erythrophleum ivorense, Euphorbia hirta, Garcinia kola, Nicotiana tabaccum, Ocimum basilicum, Pausinystalia yohimbe, Rauvolfia vomitoria, Solanum lycopersicum, Solanum nigrum, Solanum tuberosum, Voacanga africana, Voacanga thouarsii, Zingiber officinale.

Les espèces exotiques sont: Acanthus montanus, Ageratum conyzoides, Aloe vera, Allium sativum, Apium graveolens, Amaranthus hybridus, Ananas comosus, Areca catechu, Argemone mexicana, Borreria verticillata, Brassica oleracea, Camellia sinensis, Cannabis sativa, Carica papaya, Catharanthus roseus, Citrus aurantifolia, Chromolaena odorata, Coffea arabica, Coffea robusta, Combretum micranthum, Cymbopogon citratus, Dissotis rotundifolia, Eucalyptus globulus, Hibiscus esculentus, Hibiscus sabdarifa, Holarrhena floribunda, Kalanchoe crenata, Mangifera indica, Melissa officinalis, Myristica fragrans, Panax ginseng, Panax quinquerfolium, Passiflora incarnata, Persea Americana, Petroselinum sativum, Phyllathus amarus, Psidium guajava, Solanum melongena, Senna alata, Theobroma cacao, Thymus vulgaris, Zea mays.

Le ravitaillement se fait dans les champs, les forêts secondarisées, les jardins de cases et vergers, les jachères et marchés. Les feuilles, les écorces et les racines sont les parties les plus utilisées dans les recettes pour le traitement de diverses maladies. La voie orale étant le mode d'administration par excellence des dites recettes.

hétérocyclique. Ce sont des substances douées d'une grande activité biologique, même à faibles doses. Ils apparaissent dans les plantes, soit sous forme libre, soit sous forme d'un sel, soit comme $\mathrm{N}$-oxide. Les proto-alcaloïdes qui sont des amines simples, dont l'azote n'est pas inclus dans un hétérocycle. Ils dérivent aussi d'acides aminés. Les pseudo-alcaloïdes ne sont pas dérivés d'acides aminés. Ils peuvent cependant être indirectement liés à la voie des acides aminés par l'intermédiaire d'un de leurs précurseurs, ou d'un de leurs postcurseurs (dérivés). Les plantes de la famille des Solanaceae, des Apocynaceae et des Rubiaceae sont pour la plupart des plantes riches en divers alcaloïdes (Debray et al., 1971). Les alcaloïdes interviennent dans les mécanismes de défense des 
plantes contre les herbivores. La solanine, la caféine, chaconine, l'aconitine sont par exemple des alcaloïdes présents dans les graines, les feuilles et les fruits de différentes plantes où elles agissent comme des défenseurs naturels. En effet, elles sont toxiques pour les insectes, paralysant ou tuant ceux qui s'en nourrissent (Trematerre et Sciarretta, 2001 ; Bouchelta et al., 2005). En médecine, les alcaloïdes sont utilisés comme antalgiques majeurs (morphine), antipaludéen (quinine), pour combattre l'excès d'acide urique (colchicine), comme substance paralysante (curare, caféine), comme poisons (strychnine, nicotine), comme stupéfiants (cocaïne, mescaline), comme cholinergique (pilocarpine) ou comme anticancéreux (vinblastine, vincristine). Ils agissent directement sur le système nerveux avec des effets sur la conscience et la motricité. L'action sur le système nerveux peut aller jusqu'à une action antispasmodique, mydriatique, anesthésique locale ou analgésique et narcotique.

Les espèces du genre Cinchona contiennent des alcaloïdes du Groupe des quinoléines et des alcaloïdes indoliques comme la cinchonamine (Bruneton, 2009). Tous ces alcaloïdes sont réputés avoir des effets prononcés contre Plasmodium falciparum. De même, l'écorce de Alstonia boonei, les feuilles de Chromolaena odorata et Carica papaya contiennent également des alcaloïdes indoliques d'où leurs intérêts dans la lutte antipaludique. N'Guessan et al. (2009) signalaient déjà l'importance des alcaloïdes indoliques de Chromolaena odorata dans cette lutte. Toutefois, les alcaloïdes indoliques possèdent un effet inférieur à celui des alcaloïdes quinoléines dans la lutte antimalarique mais n'ont pas d'effets secondaires (comme le bourdonnement d'oreilles) (Souleymane, 2005). Nicotiana tabaccum et Capsicum annuum régulièrement prescrits contre les vers intestinaux contiennent respectivement des alcaloïdes tropaniques et la capsaïne. Ces alcaloïdes utilisées contre les maux de ventre sont réputés avoir des effets contre les spasmes gastro-intestinaux et des effets laxatifs. Ils accroissent le péristaltisme intestinal (Nacoulma, 1996 ; N'Guessan et al., 2009). En outre, Capsicum frutescens prescrit contre les douleurs lombaires contient la capsaïne dont N'Guessan et al. (2009) ont dit avoir des propriétés anti-rhumatismales. Les

\section{REFERENCES}

Aubry P. 2012. Intoxications par les plantes toxiques dans les zones tropicales et inter tropicales. Médecine tropicale. 11p.

Adjanohoun E, Cusset G, Issa LO, Keita A, Lebras M, Lejoly J, Waechter P. 1989. Banque de données de médecine traditionnelle et de pharmacopée (pharmel). Notice pour la récolte et l'entrée des données, ACCT, Paris. alcaloïdes pyrrolizidiniques de Hibiscus esculentus et indoliques (agroclavine) de Rauvolfia vomitoria exercent un effet ocytocique et sont donc utiles pour faciliter les accouchements (N'Guessan et al., 2009). De plus, Rauvolfia vomitoria prescrit pour le traitement des œdèmes des pieds contient la punarnavine (Kerharo et Adam, 1974) qui provoque une augmentation marquée et persistante de la pression sanguine avec une forte diurèse, par action sur l'épithélium rénal. En outre, la réserpine de Rauvolfia vomitoria possède des propriétés sédatives. Cette drogue est utilisée, pour traiterles états d'anxiété et les patients perturbés mentalement d'où son emploi empirique, dans le traitement des crises d'épilepsie (N'Guessan et al., 2009). L'effet contre la migraine d'Ageratum conyzoides serait le fait des alcaloïdes, probablement de type ergotamine (Frantisek et al., 1973). La caféine présente dans Cola acuminata, Coffea robusta, Coffea arabica, Camellia sinensis, Theobroma cacao a des effets antagonistes compétitifs des récepteurs de l'adénosine. C'est un neuromodulateur limitant la libération des principaux neurotransmetteurs excitateurs. C'est ainsi qu'elle entraine l'accélération du rythme cardiaque avec possibilité de palpitations, la stimulation du système nerveux central avec augmentation de la vigilance et parfois de l'anxiété, la broncho-dilatation, ou plus précisément effet antagoniste de la bronchoconstriction chez l'asthmatique où l'adénosine paraît jouer un rôle prépondérant (Debry, 1994). Les propriétés de Phyllanthus amarus, de Myristica fragrans et d'Annona muricata contre les brûlures d'estomac seraient dues à la présence d'alcaloïdes qui réduiraient la douleur et l'acidité de l'estomac, chez les patients souffrant des ulcères gastriques. La yohimbine, alcaloïde indoloterpénique de l'écorce de Pausinystalia yohimbe est considérée comme étant l'un des seuls aphrodisiaques naturels connus de l'homme et aussi le plus puissant. Elle a un effet antagoniste compétitif sur les récepteurs adrénergiques et provoque une forte stimulation psychique, une intensification des émotions sexuelles, un léger changement dans les perception pouvant provoquer de faibles hallucinations, et quelques fois des érections spontanées chez le mâle (Aubry, 2012).

Adjanohoun E, Cusset G, Issa L O, Keita A, Lebras M, Lejoly J. 1994. Banque de données de médecine traditionnelle et de pharmacopée (Pharmel). Notice pour la collecte et l'entrée des données, seconde édition. A.C.C.T., Paris.

Adjanohoun E, Aboubakar N, Dramane K, Ebot ME, Ekpere JA, Enow-Orock EG, Focho D, Gbile 
ZO, Kamanyi A, KamsuKom J, Keita A, Mbenkum T, Mbi CN, Mbiele AL, Mbome IL, Mubiru NK, Nancy WL, Nkongmeneck B, Satabie B, Sofowora A, TamzeV, Wirmum CK. 1996. Contribution to ethnobotanical and floristical studies in Cameroon. CSTR/OUA.

Adjanohoun E. 2000. La biodiversité face au développement des industries pharmaceutiques africaines. In : Réseau des " espèces ligneuses médicinales", EyogMatig O, Adjanohoun E, de Souza S et Sinsin B (eds). Compte rendu de la première réunion du réseau tenue 15-17 décembre 1999 à la station IITA Cotonou, Bénin, 88103.

Akroum S. 2011. Étude Analytique et Biologique des Flavonoïdes Naturels. Thèse de Doctorat, Université Mentouri de Constantine, Algérie. $113 p$.

Aniszewski T. 2007. Alkaloids - Secrets of Life, Alkaloid Chemistry, Biological significance, Applications and Ecological Role, Elsevier.

Awono A, Manirakiza D, Ingram V. 2009. Mobilisation et renforcement des capacités des petites et moyennes entreprises impliquées dans la filière des produits forestiers non ligneux en Afrique Centrale. CIFOR: Cameroun. http: camerounforêt. com/fr/bibliotheque/10666.

Baytop T. 1999. Therapy with plants in Turkey, past and present, second edition. Nobel Tip Kitapevi, Istanbul (in Turkish).

Béllé BJ. 1992. Lexique des essences des forêts denses centrafricaines. Ministère des eaux, forêts, Pêche et du Tourisme.

Bitsindou M. 1997. Enquêtes sur la phytothérapie traditionnelle à Kindamba et Odzala (Congo) et analyse des convergences d'usage des plantes médicinales en Afrique Centrale. Thèse de doctorat, université libre de Bruxelles, Belgique. 482p.

Biyiti LF, Meko'o DJL, Tamzc V, Amvam Zollo PH. 2004. Recherche de l'activité antibactérienne de quatre plantes médicinales camerounaises. Pharm. Med. Trad. Afr., 13 : $11-20$

Bouchelta A, Boughdad A, Blenzar A. 2005. Effets biocides des alcaloïdes, des saponines et des flavonoïdes extraits de Capsicum frutescens L. (Solanaceae) sur Bemisia tabaci (Gennadius) (Homoptera : Aleyrodidae) Biotechnologie, Agronomie, Société et Environnement. 9p.

Bruneton J. 2009. Pharmacognosie - Phytochimie, plantes médicinales, $4^{e}$ éd., revue et augmentée, Paris, Tec \& Doc - Éditions médicales internationales. 1288p.

Debray M, Jacquemin H, Razafindrambao R. 1971. Contribution à l'inventaire des plantes médicinales de Madagascar. Travaux et Documents de l'ORSTOM, 8: 1-150.

Debry G. 1994. Le café et la santé, 200-211.

Dibong SD, MpondoMpondo E, Ngoye A, Kwin NF, Betti JL. 2011a. Ethnobotanique et phytomédecine des plantes médicinales vendues sur les marchés de Douala, Cameroun. Journal of Applied Biosciences, 37: 2496-2407.

Dibong SD, MpondoMpondo E, Ngoye A, Kwin NF. 2011c. Plantes médicinales utilisées par les populations bassa de la région de Douala au Cameroun. International Journal of Biological and Chemical Sciences, 5: 1105-1117.

Din N, Saenger P, Priso RJ, Dibong SD, Amougou A. 2008. Logging activities in mangrove forests: A case study of Douala Cameroon. African Journal of Environmental Science and Technology, 2: 22-30.

Din N, MpondoMpondo E, Dibong SD, Kwin NF, Ngoye A. 2011. Inventory and Identification of plants used in the treatment of diabetes in Douala town (Cameroon). European Journal of Medicinal Plants, 1: 60-73.

Frantisek S, Stary F, Jirasek V. 1976. Plantes médicinales. Edition Lidis. 247p.

Kerharo J, Adam JG. 1974. La pharmacopée sénégalaise traditionnelle. Plantes médicinales et toxiques, Vigot frères, Paris. $1007 p$.

Letouzey R. 1970. Manuel de botanique forestière, Afrique Tropicale. Tome 2, CTFT, Sainte Marie. France.

Letouzey R. 1985. Carte phytogéographique du Cameroun au $1 / 500.000 \mathrm{n}^{\circ}$ 4. IRA-ICIV, Université de Toulouse. 240p.

Mpondo Mpondo E, Dibong SD. 2012. Traditional knowledge on medicinal plants use by ethnic communities in Douala, Cameroon. European Journal of Medicinal Plants 2: 159-176.

Mpondo Mponde E, Dibong SD, Ladoh YCF, Priso RJ, Ngoye A. 2012. Les plantes à phénols utilisées par les populations de la ville de Douala. Journal of Animal \& Plant Sciences, 15: 2083-2098.

Nacoulma-Ouédraogo O. 1996. Plantes médicinales et pratiques médicales traditionnelles au Burkina Faso: cas du Plateau central, Thèse de Doctorat ès Sciences Naturelles, Université de Ouagadougou, (Burkina-Faso). 605 p. 
N'Guessan K, Kadja B, Zirihi GN, Traoré D, Aké-assi L. 2009. Screening phytochimique de quelques plantes médicinales ivoiriennes. Sciences \& Nature, 6: 1-15.

Organisation Mondiale de la Santé (OMS). 1996. Classification statistique internationale des maladies et des problèmes de santé connexes, 10ème révision, vol. 3, OMS, Genève.

Organisation Mondiale de la Santé (OMS). 2002. Stratégie de l'OMS pour la médecine traditionnelle pour 2002-2005, Genève. 78p.

Priso RJ, Nnanga JF, Etame J, Din N, Amougou A. 2011. Les produits forestiers non ligneux d'origine végétale : valeur et importance dans quelques marchés de la région du LittoralCameroun. Journal of Applied Biosciences, 40: $2715-2726$.

Schnell R. 1960. Techniques d'herborisation et de conservation des plantes dans les pays tropicaux. J. Agric. Trop. Bot. Appl., 7: 1-48.

Souleymane F. 2005. Exploration biochimique sur le pouvoir immunogène de trois plantes en Côte d'ivoire Alstonia boonei, Mitragyna ciliata, Terminalia catappa. Thèse de Doctorat, Université de Bamako, Mali. 102p.

Thirakul S. 1995. Manuel de dendrologie des forêts denses de Centrafrique. ACDI.

Trematerra P, Sciarretta A. 2002. Activity of chilli, Capsicum annuum L. var. acuminatum, on stored product insects Oryzaephilus surinamensis (L.), Sitophilusoryzae (L.) and Tribolium castaneum (Herbst). IOBC/wprs Bull., 25: 177-182.

World Health Organisation (WHO). 2010. International Statistical Classification of Diseases and Related Health Problems. 10th Revision. Instruction manual vol. 22010 Edition.WHO, Geneva. 199p.

Zirihi GN, Grellier P, Guédé-Guina F, Bodo B, Lengo M. 2005. Isolation, Characterisation and antiplasmodial activity of steroidal alkaloids from Funtumia elastic (Preuss) Stapf. Biorganic and Medicinal Chemistry Letters, 15: 2637-2640.

Zirihi GN. 2006. Études botanique, pharmacologique et phytochimique de quelques plantes Médicinales anti-paludiques et/ou immunogènes utilisées chez les Bété du Département d'Issia, dans l'ouest de la Côte d'Ivoire. Thèse de Doctorat d'État, Université de Cocody Abidjan, UFR Biosciences. 126p.

Zirihi GN, Datté JY, Kra-Adou KM, Grellier P. 2007. Phytochemical and pharmacological studies of the alcoholic extract (MFA) of Fagara macrophylla (Oliv.) Engl. (Rutaceae) : the chemical structure of the active compound inducing antipaludic activity. Journal of Chinese Clinical Medicine, 2 : 205-210. 
Tableau 1. Quelques usages des plantes à alcaloïdes de la ville de Douala.

\begin{tabular}{|c|c|c|c|c|c|}
\hline Nom scientifiques & $\begin{array}{c}\text { Noms } \\
\text { communs }\end{array}$ & Modes de préparation & $\begin{array}{l}\text { Parties } \\
\text { utilisées }\end{array}$ & Posologie & Alcaloïdes \\
\hline $\begin{array}{l}\text { Adansonia digitata Linn. } \\
\text { (Bombacaceae) }\end{array}$ & Baobab & $\begin{array}{l}\text { anti diarrhéique: sécher le fruit, le débarrasser de ses } \\
\text { graines et mélanger dans de l'eau ou du lait }\end{array}$ & Pulpe & boire au repas & Adansonine \\
\hline $\begin{array}{l}\text { Ageratum conyzoïdes } \\
\text { Linn. (Asteraceae) }\end{array}$ & $\begin{array}{l}\text { roi des } \\
\text { herbes }\end{array}$ & $\begin{array}{l}\text { 1. Blessures et morsures : extraire le jus des feuilles } \\
\text { 2. Migraines : extraire le jus des feuilles et boire }\end{array}$ & Feuilles & $\begin{array}{l}\text { Appliquer le jus sur les } \\
\text { parties concernées }\end{array}$ & $\begin{array}{l}\text { Licopsamine, } \\
\text { Ergotamine, } \\
\text { alcaloides } \\
\text { Pyrrolidiniques }\end{array}$ \\
\hline $\begin{array}{l}\text { Allium cepa Linn. } \\
\text { (Liliaceae) }\end{array}$ & Oignon & $\begin{array}{l}\text { Hypertension artérielle : infusion des feuilles et du } \\
\text { bulbe }\end{array}$ & Feuilles, bulbe & $\begin{array}{l}\text { Boire dans un verre d'eau } \\
\text { matin, midi et soir }\end{array}$ & $\begin{array}{l}\text { glucokinine, alliine, } \\
\text { allicine }\end{array}$ \\
\hline $\begin{array}{l}\text { Allium sativum Linn. } \\
\text { (Liliaceae) }\end{array}$ & ail & $\begin{array}{l}\text { 1. Douleurs d'estomac : découper les gousses en } \\
\text { petites tranches et laisser macérer ; } \\
\text { 2. Hypertension : laver les gousses et laisser macérer ; } \\
\text { 3. Excès de cholestérol : éplucher les gousses et } \\
\text { laisser macérer ; } \\
\text { 4. Antimycosiques, antibactérien, antibiotique : macérer } \\
1 / 2 \text { poignée d'ail dans } 1 / 2 \text { litre de vinaigre filtrer et } \\
\text { conserver dans les bouteilles bien bouchées pendant } \\
10 \text { jours ; } \\
5 . \text { fièvre typhoïde : écraser une poignée d'ail et } 2 \\
\text { oignons, porter le mélange à ébullition dans } 2 \text { litres de } \\
\text { lait non sucré et laisser refroidir ; } \\
\text { 6. Maux d'estomac : éplucher les gousses et les } \\
\text { découper en petits morceaux et mettre dans de l'eau; } \\
\text { 7. Mal d'oreille : presser quelques gouttes d'ail et } \\
\text { obtenir un jus d'ail. }\end{array}$ & Gousses & $\begin{array}{l}\text { 1. Boire matin, midi et soir ; } \\
\text { 2. Mâcher ; } \\
\text { 3. Manger les gousses ; } \\
\text { 4. Boire dans un verre } \\
\text { d'eau matin et soir ; } \\
\text { 5. Boire matin et soir dans } \\
\text { un verre d'eau pendant } \\
\text { deux semaines; } \\
\text { 6. Boire dans un verre } \\
\text { d'eau ; } \\
\text { 7. Mettre quelques gouttes } \\
\text { dans l'oreille à l'aide d'une } \\
\text { pipette. }\end{array}$ & Alliine, Allicine \\
\hline $\begin{array}{l}\text { Aloe vera Linn. } \\
\text { (Asparagaceae) }\end{array}$ & Aloe vera & $\begin{array}{l}\text { 1. Cholestérol : mélanger à de l'eau le gel obtenu à } \\
\text { partir des feuilles macérées débarrassées au préalable } \\
\text { des épines; }\end{array}$ & Feuilles & $\begin{array}{l}\text { 1. Boire dans } 1 / 2 \text { verre d'eau } \\
\text { matin et soir pendant trois } \\
\text { jours ; }\end{array}$ & $\begin{array}{l}\text { Aloïne, Aloetine, } \\
\text { barbaloïne, } \\
\text { Hydroanthroquinone }\end{array}$ \\
\hline
\end{tabular}




\begin{tabular}{|c|c|c|c|c|c|}
\hline & & $\begin{array}{l}\text { 2. Brûlures : nettoyer les feuilles dans de l'eau salée, } \\
\text { enlever les épines, diviser les feuilles en deux parties } \\
\text { égales et gratter les faces intérieures pour extraire le } \\
\text { mucilage ; } \\
\text { 3. Paludisme : nettoyer les feuilles, enlever les épines, } \\
\text { diviser les feuilles en deux parties égales et gratter les } \\
\text { faces intérieures pour extraire le mucilage ; } \\
\text { 4. Épuration de l'organisme : macération pendant au } \\
\text { moins deux heures des feuilles débarrassées des } \\
\text { épines ; } \\
\text { 5. Anti inflammatoire: macération pendant au moins } \\
\text { deux heures des feuilles débarrassées des épines ; } \\
\text { 6. Purgatif et vermifuge dans les infections digestives } \\
\text { et biliaires: décoction (prendre une demi feuille, enlevé } \\
\text { les piquants et la peau, découper en petits morceaux et } \\
\text { mettre dans } 1 \text { litre d'eau. Porter le mélange à ébullition } \\
\text { pendant } 20 \text { minutes ; } \\
\text { 7. Chute de cheveux: extraire le gel gluant des feuilles ; } \\
\text { 8. Diabète: découper les feuilles en petits morceaux et } \\
\text { porter à ébullition dans un litre d'eau et extraire le gel } \\
\text { gluant des feuilles; } \\
\text { 9. Brûlures: macérer des morceaux de feuilles dans } \\
\text { l'eau pendant } 4 \text { heures et extraire le gel gluant des } \\
\text { feuilles ; } \\
\text { 10. Éruption cutanée : macérer des morceaux de } \\
\text { feuilles dans l'eau pendant } 4 \text { heures et extraire le gel } \\
\text { gluant des feuilles; } \\
\text { 11. vomissement: macérer des morceaux de feuilles } \\
\text { dans de l'eau ; } \\
\text { 12. Amincissant: macérer des morceaux de feuilles } \\
\text { dans de l'eau ; } \\
\text { 13. Constipation: Bouillir une } 1 / 2 \text { feuille coupée en } \\
\text { morceaux dans } 1 \text { litre d'eau pendant } 20 \text { minutes ; } \\
\text { 14. Eczéma: extraire le mucilage. }\end{array}$ & & $\begin{array}{l}\text { 2. Appliquer le mucilage sur } \\
\text { les parties brulées ; } \\
\text { 3. Diluer avec l'eau et } \\
\text { boire; } \\
\text { 4. Diluer avec l'eau et } \\
\text { boire ; } \\
\text { 5. Diluer avec l'eau et } \\
\text { boire ; } \\
\text { 6. Boire dans une tasse le } \\
\text { soir pour obtenir une action } \\
\text { le matin; } \\
\text { 7. Masser du cuir chevelure } \\
\text { deux fois par jour ; } \\
\text { 8. Boire une tasse du gel } 1 \\
\text { fois par jour et pour les } \\
\text { diabétiques } 1 \text { cuillère à } \\
\text { soupe; } \\
\text { 9. Appliquer le gel sur la } \\
\text { peau; } \\
\text { 10. Boire } 1 \text { cuillérée à } \\
\text { soupe toutes les } 6 \text { heures ; } \\
\text { 11. Boire une demi-tasse } \\
\text { deux fois par jour ; } \\
\text { 12.Se purger } \\
\text { régulièrement; } \\
\text { 13. Boire } 1 \text { tasse le soir et } \\
\text { par jour ; } \\
\text { 14. Appliquer sur les parties } \\
\text { malades. }\end{array}$ & $\begin{array}{c}\text { et anthraquinone, } \\
\text { Résine, } \\
\text { Anthracénoside, } \\
\text { Nicotine, Mucilages, } \\
\text { aloïne, } \\
\text { anthraquinone, , } \\
\text { cholinem Stachydrine }\end{array}$ \\
\hline Alstonia boonei De Wild. & Emien & Antipaludéen: décoction des écorces & Ecorces & & \\
\hline
\end{tabular}




\begin{tabular}{|c|c|c|c|c|c|}
\hline (Apocynaceae) & $\begin{array}{c}\text { Ekuk } \\
\text { (éwondo) }\end{array}$ & & & $\begin{array}{l}\text { Boire un verre d'eau matin } \\
\text { et soir pendant six jours }\end{array}$ & $\begin{array}{l}\text { Echitamine, } \\
\text { échitamidine }\end{array}$ \\
\hline $\begin{array}{l}\text { Amaranthus hybridus L. } \\
\text { (Amaranthaceae) }\end{array}$ & $\begin{array}{c}\text { Ewole a } \\
\text { mole }\end{array}$ & $\begin{array}{l}\text { Erythème fessier chez le nourrisson: macérer les } \\
\text { feuilles et extraire le jus, ajouter } 1 / 4 \text { du fruit de bongo } \\
\text { (bassa) écrasé dans le jus, tamiser et porter à ébullition }\end{array}$ & Feuilles & $\begin{array}{l}\text { Faire deux purges à } \\
\text { intervalles de quelques } \\
\text { heures }\end{array}$ & $\begin{array}{c}\text { Alcaloïdes } \\
\text { quinolizidiniques }\end{array}$ \\
\hline $\begin{array}{l}\text { Ananas comosus (L.) } \\
\text { Merr. (Bromeriaceae) }\end{array}$ & Ananas & Excès de poids: Faire une décoction de l'épicarpe & Epicarpe & $\begin{array}{l}\text { Boire dans un verre d'eau } \\
\text { matin et soir }\end{array}$ & bromélaine \\
\hline $\begin{array}{l}\text { Annona muricata Linn. } \\
\text { (Annonaceae) }\end{array}$ & Corossol & $\begin{array}{l}\text { 1. Mal d'estomac : Bouillir les feuilles dans } 1 \text { litre d'eau } \\
\text { ou consommer le fruit ; } \\
\text { 2. cancer : Faire un broyat de la pulpe et des graines ; } \\
\text { 3. Fatigue, insomnie, toux, dysenterie : Bouillir les } \\
\text { feuilles dans } 1 \text { litre d'eau pendant } 20 \text { minutes ; } \\
\text { 4. Tension artérielle : Bouillir les feuilles dans } 1 \text { litre } \\
\text { d'eau pendant } 1 \mathrm{~h} \text {; } \\
5 \text {. Insomnie : Chauffer } 1 \text { litre d'eau et y infuser les } \\
\text { feuilles. }\end{array}$ & $\begin{array}{l}\text { Feuilles, fruits, } \\
\text { graines, pulpe }\end{array}$ & $\begin{array}{l}\text { 1. Boire un verre d'eau } \\
\text { matin et soir ; } \\
\text { 2. Boire un verre le matin et } \\
\text { le soir pendant tout le } \\
\text { traitement; } \\
\text { 3. Boire un verre le matin et } \\
\text { le soir pendant tout le } \\
\text { traitement; } \\
\text { 4. Boire un verre le matin et } \\
\text { le soir pendant tout le } \\
\text { traitement; } \\
\text { 5. Boire un verre le matin et } \\
\text { le soir pendant tout le } \\
\text { traitement. }\end{array}$ & $\begin{array}{l}\text { Liriodenine, } \\
\text { Athérospermine, } \\
\text { Alcaloïdes sédatifs } \\
\text { (liriodénine et } \\
\text { athérospermine) }\end{array}$ \\
\hline $\begin{array}{l}\text { Apium graveolens Linn. } \\
\text { (Apiaceae) }\end{array}$ & céleri & $\begin{array}{l}\text { 1. maladies chroniques : mélanger du jus de céleri et } \\
\text { du jus de carottes; } \\
\text { 2. Cystite, infection urinaire, bronchite, arthrite, goutte : } \\
\text { Tisanes de graines (infusion de graines de céleri). }\end{array}$ & $\begin{array}{c}\text { Feuilles, graines } \\
\text { et branches }\end{array}$ & $\begin{array}{l}\text { 1. Prendre une tasse par } \\
\text { jour ; } \\
\text { 2. Prendre une tasse par } \\
\text { contenant } 1,5 \mathrm{~g} \text { de graines } \\
\text { de céleri. }\end{array}$ & Para alcaloïdes \\
\hline $\begin{array}{l}\text { Areca catechu L. } \\
\text { (Arecaceae) }\end{array}$ & $\begin{array}{l}\text { Palmier } \\
\text { Bétel }\end{array}$ & Stimulant, tonique : Ecraser et macérer les noix & Noix & $\begin{array}{l}\text { Consommer } 1 \text { cuillère à } \\
\text { soupe matin et soir }\end{array}$ & Arécaïne, Arécoline \\
\hline $\begin{array}{l}\text { Argemone mexicana L. } \\
\text { (Papaveraceae) }\end{array}$ & $\begin{array}{c}\text { Pavot } \\
\text { mexicain }\end{array}$ & $\begin{array}{l}\text { 1. Céphalées : Bouillir ensemble les fleurs et les } \\
\text { feuilles dans } 1 \text { litre d'eau; } \\
\text { 2. Infections pulmonaires, toux, migraines : Bouillir les } \\
\text { feuilles dans } 1 \text { litre d'eau; } \\
\text { 3. verrues; herpès ; ophtalmies : Mettre } 1 \text { poignée de }\end{array}$ & $\begin{array}{l}\text { Fleurs, feuilles, } \\
\text { graines }\end{array}$ & $\begin{array}{l}\text { 1. Boire } 1 \text { verre de décocté } \\
\text { matin et soir ; } \\
\text { 2. Boire } 1 \text { verre de décocté } \\
\text { matin et soir ; } \\
\text { 3. Faire une application }\end{array}$ & Alcaloïde toxique \\
\hline
\end{tabular}




\begin{tabular}{|c|c|c|c|c|c|}
\hline & & $\begin{array}{l}\text { feuilles fraîches dans } 1 \text { litre de vin de madère, couvrir } \\
\text { et laisser macérer pendant } 12 \text { heures. }\end{array}$ & & $\begin{array}{l}\text { locale de la préparation } 2 \\
\text { fois par jour jusqu'à entière } \\
\text { guérison. }\end{array}$ & \\
\hline $\begin{array}{l}\text { Baillonella toxisperma } \\
\text { Pierre. (Sapotaceae) }\end{array}$ & Moabi & $\begin{array}{l}\text { Maux de reins ; douleurs dentaires ; rachitisme ; } \\
\text { infections vaginales, infections des voies respiratoires } \\
\text { et digestives : Mélanger une décoction d'écorces dans } \\
1 \text { litre d'eau }\end{array}$ & Ecorces & $\begin{array}{l}\text { Boire } 1 \text { verre de décocté } \\
\text { matin et soir }\end{array}$ & yahimbine \\
\hline $\begin{array}{l}\text { Borreria verticillata (L.) } \\
\text { G.F.W. Meyer. } \\
\text { (Rubiaceae) }\end{array}$ & $\begin{array}{l}\text { Borreria } \\
\text { verte }\end{array}$ & $\begin{array}{l}\text { Plaies, furoncles, panaris, lèpre et eczémas : Faire une } \\
\text { décoction de feuilles fraîches pilées }\end{array}$ & & $\begin{array}{l}\text { Appliquer localement } \\
\text { jusqu'à entière guérison }\end{array}$ & $\begin{array}{l}\text { Borrevine, borrerine, } \\
\text { isoborreverine. }\end{array}$ \\
\hline $\begin{array}{l}\text { Brassica oleracea Linn. } \\
\text { (Brassicacea) }\end{array}$ & Chou & $\begin{array}{l}\text { 1. Cicatrisation des plaies : Laver la feuille à l'eau } \\
\text { bouillante et écraser la nervure médiane, faire macérer } \\
\text { dans l'eau boriquée pendant } 12 \text { heures; } \\
\text { 2. Angine, maux de gorge : Gargarisme ; } \\
\text { 3. Douleurs musculaires : Faire chauffer de l'eau avec } \\
\text { une bonne quantité de gros sel, ajouter les feuilles de } \\
\text { choux pour qu'elles ramollissent ; } \\
\text { 4. Ulcères gastriques: Verser deux cuillerées à soupe } \\
\text { de feuilles de chou finement hachées dans une demi- } \\
\text { tasse d'eau bouillante sur. }\end{array}$ & Feuilles & $\begin{array}{l}\text { 1. Appliquer localement et } \\
\text { maintenir avec un } \\
\text { bandage ; } \\
\text { 2. Gargarisme ; } \\
\text { 3. Appliquer localement et } \\
\text { maintenir avec un } \\
\text { bandage. } \\
\text { 4. Boire } 1 / 2 \text { tasse deux à } \\
\text { trois fois par jour. }\end{array}$ & Brocoline \\
\hline $\begin{array}{l}\text { Camellia sinensis (L.) } \\
\text { Kuntze (Theaceae) }\end{array}$ & thé & $\begin{array}{l}\text { 1. Infections digestives : Faire une infusion forte de thé } \\
\text { 2. Stimule le système nerveux : Faire une infusion }\end{array}$ & Feuilles & Boire 1 verre infusé & Cafeïne \\
\hline $\begin{array}{l}\text { Cannabis sativa L. } \\
\text { (Cannabinaceae) }\end{array}$ & Chanvre & $\begin{array}{l}\text { Taux de cholestérol élevé : Laisser infuser pendant } 10 \\
\text { minutes une cuillère à soupe de chanvre dans } 1 \text { litre } \\
\text { d'eau }\end{array}$ & Fleurs, fruits & Boire 2 ou 3 tasses par jour & Tétrahydrocannabinol \\
\hline $\begin{array}{l}\text { Capsicum annuum L. } \\
\text { (Solanaceae) }\end{array}$ & Gros piment & $\begin{array}{l}\text { Constipation et infections intestinales : Prendre } \\
\text { quelques feuilles de ndolè, les broyer dans un gobelet } \\
\text { d'eau et ajouter quelques graines de piment } \\
\text { (légèrement écrasées) et bien mélanger }\end{array}$ & $\begin{array}{l}\text { Fruits, feuilles, } \\
\text { graines, }\end{array}$ & Prendre 2 ou 3 poires & $\begin{array}{l}\text { Capsaïcine, } \\
\text { Alcaloïdes non } \\
\text { hétérocycliques. }\end{array}$ \\
\hline $\begin{array}{l}\text { Capsicum frutescens } \\
\text { Linn. (Solanaceae) }\end{array}$ & Petit piment & $\begin{array}{l}\text { 1. Douleurs lombaires : Epicer les repas avec la poudre } \\
\text { de piment; } \\
\text { 2. Perturbations du flux sanguin : Verser } 1 / 2 \text { cuillerée } \\
\text { de piment en poudre dans } 1 \text { tasse d'eau bouillante et } \\
\text { laisser reposer pendant } 10 \text { minutes. }\end{array}$ & Fruits & $\begin{array}{l}\text { 1. Consommer vos repas ; } \\
\text { 2. Mélanger } 1 \text { cuillerée à } \\
\text { soupe de cette infusion et } \\
\text { boire selon les besoins. }\end{array}$ & $\begin{array}{l}\text { Capsaicine, } \\
\text { Pipérine, capsicine, } \\
\text { alpha et béta, }\end{array}$ \\
\hline Carica papaya Linn. & papayer & 1. Constipation : Mélanger les graines dans 1 litre & Fruits verts, & 1. Boire une tasse matin et & Vitamine $A, B, C, E$, \\
\hline
\end{tabular}




\begin{tabular}{|c|c|c|c|c|c|}
\hline (Caricaceae) & & $\begin{array}{l}\text { d'eau sucrée ; } \\
\text { 2. Dysenterie amibienne : Sécher les pépins, les } \\
\text { moudre jusqu'à l'obtention d'une poudre homogène et } \\
\text { faire bouillir. } \\
\text { 3. Vers intestinaux : Bouillir en mélange les feuilles et } \\
\text { les pépins ; } \\
\text { 4. Céphalées : Piler les racines et les feuilles et extraire } \\
\text { le jus ; } \\
\text { 5. Jaunisse, bronchite et trachéite : Déterrer quelques } \\
\text { racines, bien les laver et découper dans une } \\
\text { casserole ; ajouter de l'eau et du citron coupé en } \\
\text { tranches. Faire bouillir pendant environ } 40 \text { minutes et } \\
\text { laisser refroidir ; } \\
\text { 6. Troubles gastriques et intestinaux : Verser } 75 \mathrm{~g} \text { de } \\
\text { feuilles dans un } 1 / 2 \text { litre d'eau et laisser infuser } 10 \\
\text { minutes; } \\
\text { 7. Paludisme : Bouillir les feuilles sèches dans } 1 \text { litre } \\
\text { d'eau pendant } 25 \text { minutes et y ajouter } 3 \text { cuillérées de } \\
\text { miel ; } \\
\text { 8. Allergies : Bouillir une tasse d'eau contenant } 30 \mathrm{~g} \\
\text { de graines ou du fruit dans pendant } 5 \text { minutes ; } \\
\text { 9. maladies vénériennes : Ecraser les feuilles de } \\
\text { papayer et les diluer dans } 1 \text { litre d'eau }\end{array}$ & $\begin{array}{l}\text { graines, feuilles, } \\
\text { racine, latex }\end{array}$ & $\begin{array}{l}\text { soir ; } \\
\text { 2. Boire un verre trois fois } \\
\text { par jour ; } \\
\text { 3. Boire } 1 \text { verre de décocté } \\
\text { matin et soir ; } \\
\text { 4. Boire } 1 \text { verre de } \\
\text { décocté ; } \\
\text { 5. Boire } 1 \text { verre de cette } \\
\text { infusion matin et soir } \\
\text { jusqu'à guérison complète ; } \\
6 \text {. Boire } 1 / 2 \text { tasse } 2 \text { fois par } \\
\text { jour ; } \\
\text { 7. Boire } 3 \text { verres par jour ; } \\
8 \text {. Boire cette tasse infusée } \\
\text { à chaud; } \\
9 . \text { Boire une tasse matin et } \\
\text { soir jusqu'à guérison }\end{array}$ & $\begin{array}{c}\text { pectine, } \\
\text { papaine, carpaine, } \\
\text { chimopapaine, } \\
\text { pancréatine }\end{array}$ \\
\hline $\begin{array}{l}\text { Catharanthus roseus (L.) } \\
\text { G. Don. (Apocynaceae) }\end{array}$ & $\begin{array}{l}\text { Pervenche } \\
\text { de } \\
\text { Madagascar }\end{array}$ & $\begin{array}{l}\text { 1. Hypotension : Faire une décoction des feuilles ( } 40 \mathrm{~g} \\
\text { dans } 1 \text { litre d'eau); } \\
\text { 2. Meurtrissure, choc physique, angine, plaies : feuilles } \\
\text { brouillées } \\
\text { 3. Diabète : Infusion des feuilles ( } 40 \mathrm{~g} \text { dans } 1 \text { litre } \\
\text { d'eau); } \\
\text { 4. Vermifuge : Faire une décoction avec de l'eau } \\
\text { potable des écorces et des racines; } \\
\text { 5. Toux : Infuser } 30 \text { fleurs dans } 1 \text { litre d'eau. }\end{array}$ & $\begin{array}{c}\text { Feuilles, } \\
\text { écorces, } \\
\text { racines, fleurs }\end{array}$ & $\begin{array}{l}\text { 1. Boire } 1 \text { tasse de décocté } \\
\text { matin et soir ; } \\
\text { 2. emplâtre sur les bleus ; } \\
\text { 3. Boire } 1 \text { tasse d'infusé } \\
\text { matin et soir ; } \\
\text { 4. Boire } 1 \text { verre de décocté } \\
\text { chaud, } 3 \text { fois par jour } \\
\text { pendant } 7 \text { jours ; } \\
\text { 5. Prendre } 2 \text { cuillérées à } \\
\text { soupe } 3 \text { fois par jour. }\end{array}$ & $\begin{array}{l}\text { Vincristine, } \\
\text { vinblastine, reserpine, } \\
\text { ibogaine, yoimbine, } \\
\text { Leurosidine, } \\
\text { catharantine, } \\
\text { Strophantine, } \\
\text { ajmalicine, ajmaline }\end{array}$ \\
\hline Chromolaena odorata & Herbe du & 1. Diabète, paludisme : Faire une décoction des feuilles & & 1. Boire 1 tasse de décocté & Alcaloïdes \\
\hline
\end{tabular}




\begin{tabular}{|c|c|c|c|c|c|}
\hline $\begin{array}{l}\text { (L.) King \& H.E. Robins. } \\
\text { (Asteraceae) }\end{array}$ & Laos & $\begin{array}{l}\text { dans } 1 \text { litre d'eau ; } \\
\text { 2. Diarrhées : Pétrir les feuilles et y ajouter de l'eau. }\end{array}$ & & $\begin{array}{l}\text { matin et soir; } \\
\text { 2. Se purger. }\end{array}$ & $\begin{array}{l}\text { pyrrolizidiniques : } \\
\text { (rétronécine, } \\
\text { héliotridine, } \\
\text { platynécine) }\end{array}$ \\
\hline $\begin{array}{l}\text { Cinchona calisaya Weed. } \\
\text { (Rubiaceae) }\end{array}$ & $\begin{array}{l}\text { Quinquina } \\
\text { jaune }\end{array}$ & $\begin{array}{l}\text { 1. Paludisme : Faire bouillir les écorces dans } 1 \text { litre } \\
\text { d'eau; } \\
\text { 2. Démangeaisons et pellicules : Bouillir les racines } \\
\text { dans } 1 \text { litre d'eau; } \\
\text { 3. Desquamations : Sécher et réduire en poudre les } \\
\text { écorces ; } \\
\text { 4. Ballonnements, Dyspepsie : Mettre } 5 \mathrm{~g} \text { d'écorce } \\
\text { dans } 1 \text { litre d'eau bouillante. }\end{array}$ & $\begin{array}{l}\text { Ecorces et } \\
\text { racines }\end{array}$ & $\begin{array}{l}\text { 1. Boire } 1 \text { tasse de décocté } \\
\text { matin et soir ; } \\
\text { 2. Boire } 1 \text { tasse de décocté } \\
\text { matin et soir ; } \\
\text { 3. Appliquer la poudre au } \\
\text { lieu indiqué ; } \\
\text { 4. Boire } 1 \text { cuillerée à café } \\
\text { quatre fois par jour. }\end{array}$ & $\begin{array}{l}\text { Quinine, Quinidine, } \\
\text { Cinchonine, } \\
\text { Cinchonidine }\end{array}$ \\
\hline $\begin{array}{l}\text { Cinchona officinalis L. } \\
\text { (Rubiaceae) }\end{array}$ & $\begin{array}{l}\text { Quinquina } \\
\text { gris }\end{array}$ & $\begin{array}{l}\text { 1. Paludisme : Bouillir les écorces dans1 litre d'eau ; } \\
\text { 2. Allergies : Verser } 5 \mathrm{~g} \text { d'écorces finement coupées } \\
\text { dans } 1 \text { litre d'eau bouillante, couvrir et laisser reposer } \\
\text { pendant } 10 \text { minutes ; } \\
\text { 3. Fièvre : Sécher et réduire en poudre les écorces. }\end{array}$ & Ecorces & $\begin{array}{l}\text { 1. Boire } 1 \text { tasse de décocté } \\
\text { matin et soir ; } \\
\text { 2. Boire un } 1 / 2 \text { verre matin } \\
\text { et soir jusqu'à disparition } \\
\text { des symptômes; } \\
\text { 3. Boire } 1 \text { cuillerée à soupe } \\
\text { de la poudre diluée dans de } \\
\text { l'eau. }\end{array}$ & $\begin{array}{l}\text { Quinquine, } \\
\text { Cinchonine, } \\
\text { cinchonidinehonine }\end{array}$ \\
\hline $\begin{array}{l}\text { Cinchona pubescens } \\
\text { Vahl. (Rubiaceae) }\end{array}$ & $\begin{array}{l}\text { Quiquina } \\
\text { rouge }\end{array}$ & $\begin{array}{l}\text { Fièvre jaune : Tremper les écorces dans une assiette } \\
\text { d'eau contenant du café }\end{array}$ & écorce & $\begin{array}{l}\text { Boire dans } 1 \text { verre d'eau } \\
\text { fois par jour }\end{array}$ & quinine, tanins \\
\hline $\begin{array}{l}\text { Citrus aurantifolia } \\
\text { (Christm.) Swingle. } \\
\text { (Rutaceae) }\end{array}$ & Oranger & $\begin{array}{l}\text { 1. Digestif ; } \\
\text { 2. Diabète : Râper les écorces séchées et mettre une } \\
\text { petite cuillerée du produit dans une tasse d'eau ; } \\
\text { 3. Douleurs d'estomac : Râper les écorces séchées et } \\
\text { mettre une petite cuillerée du produit dans une tasse } \\
\text { d'eau; } \\
\text { 4. Règles irrégulières : Infuser les différentes parties de } \\
\text { la plante. }\end{array}$ & $\begin{array}{l}\text { Fruits, feuilles, } \\
\text { écorce }\end{array}$ & $\begin{array}{l}\text { 1. Consommer une orange } \\
\text { après chaque repas ; } \\
\text { 2. Boire } 1 \text { tasse par jour ; } \\
\text { 3. Boire } 1 / 2 \text { tasse à jeûne; } \\
\text { 4. Boire } 1 \text { tasse par jour. }\end{array}$ & $\begin{array}{l}\text { huiles essentielles, } \\
\text { hespéridine, } \\
\text { caroténoïdes, } \\
\text { vitamine C, } \\
\text { Caroténoïdes, } \\
\text { Hespéridine, } \\
\text { Glucoside }\end{array}$ \\
\hline $\begin{array}{l}\text { Coffea arabica L. } \\
\text { (Rubiaceae) }\end{array}$ & Café arabica & $\begin{array}{l}\text { Faiblesse ou somnolence : Dissoudre la poudre dans } \\
\text { une tasse d'eau chaude }\end{array}$ & Feuille, graines & Boire une tasse par jour & $\begin{array}{l}\text { Caféine, triméthyl, } \\
\text { xanthine }\end{array}$ \\
\hline $\begin{array}{l}\text { Coffea robusta Pierre ex } \\
\text { A. Froehner. (Rubiaceae) }\end{array}$ & Caféier & $\begin{array}{l}\text { Stimulant de système nerveux : Faire bouillir une } \\
\text { poignée de feuilles dans } 1 \text { litre d'eau ; Au moment } \\
\text { d'éteindre le feu ajouter une feuille de citronnelle et }\end{array}$ & Graine & Boire une tasse par jour & Caféine \\
\hline
\end{tabular}




\begin{tabular}{|c|c|c|c|c|c|}
\hline & & laisser infuser 5 à 10 minutes puis filtrer & & & \\
\hline $\begin{array}{l}\text { Cola acuminata } \\
\text { (P. Beauv.) Schott \& } \\
\text { Endl. (Sterculiaceae) }\end{array}$ & Kolatier & $\begin{array}{l}\text { 1. Antidépresseur et aphrodisiaque ; } \\
\text { 2. Stimule le système nerveux et assure la tonicité } \\
\text { musculaire ; } \\
\text { 3. Accouchement facile : Faire une décoction d'une } \\
\text { poignée fraîche d'écorce de kolatier et laisser } \\
\text { fermenter pendant } 12 \mathrm{~h} \text {. }\end{array}$ & $\begin{array}{l}\text { Fruit, noix et } \\
\text { écorce }\end{array}$ & $\begin{array}{l}\text { 1. Mâcher } 1 \text { à } 2 \text { noix par } \\
\text { jour; } \\
\text { 2. Mâcher } 1 \text { à } 2 \text { noix par } \\
\text { jour; } \\
\text { 3. prendre } 1 \text { verre matin et } \\
\text { soir à partir du septième } \\
\text { mois de la grossesse } \\
\text { jusqu'à l'accouchement }\end{array}$ & $\begin{array}{l}\text { Caféine, Kolatine, } \\
\text { Kolatéine, } \\
\text { théophylline, } \\
\text { Théobromine }\end{array}$ \\
\hline $\begin{array}{l}\text { Cola nitida Schott \& } \\
\text { Endl. (Sterculiaceae) }\end{array}$ & Kolatier & $\begin{array}{l}\text { Asthénie sexuelle : Sécher les noix et les écraser pour } \\
\text { obtenir une poudre et faire une infusion de poudre }\end{array}$ & Fruits et noix & $\begin{array}{l}\text { Boire } 1 \text { verre de cette } \\
\text { infusion trois fois par jour ; }\end{array}$ & $\begin{array}{c}\text { Caféine, } \\
\text { methylxanthines, } \\
\text { tanins, Théobromine, } \\
\text { Xanthines, kolanine }\end{array}$ \\
\hline $\begin{array}{l}\text { Combretum } \\
\text { micranthum G. Don } \\
\text { (Combretaceae) }\end{array}$ & Kinkeliba & $\begin{array}{l}\text { 1. Constipation et grippe : Faire une infusion de } \\
\text { feuilles ; } \\
\text { 2. Plaies : Mâcher les feuilles ; } \\
\text { 3. Malaria : Faire une décoction de feuilles, écorce, } \\
\text { racines, fruits }\end{array}$ & $\begin{array}{l}\text { Feuilles, } \\
\text { écorces, racines } \\
\text { et fruits }\end{array}$ & $\begin{array}{l}\text { 1. Boire } 1 \text { verre de cette } \\
\text { infusion matin et soir ; } \\
\text { 2. Faire une application des } \\
\text { feuilles mâchées sur la } \\
\text { plaie; } \\
\text { 3. Boire } 1 \text { verre de cette } \\
\text { décoction matin et soir } \\
\text { pendant } 3 \text { jours. }\end{array}$ & $\begin{array}{l}\text { choline, } \\
\text { starchydrine, } \\
\text { Nitrate } \\
\text { potassium }\end{array}$ \\
\hline $\begin{array}{l}\text { Costus spicatus (Jacq.) } \\
\text { Sw. (Costaceae) }\end{array}$ & Amome velu & $\begin{array}{l}\text { Affection et inflammation de l'appareil urinaire, calculs } \\
\text { rénaux : Décoction de } 5 \text { cuillères à café de tiges } \\
\text { coupées en petit morceaux dans } 1 \text { litre d'eau et bouillir }\end{array}$ & Tiges & $\begin{array}{l}\text { Boire } 3 \text { à } 4 \text { tasses de } \\
\text { décocté par jour }\end{array}$ & \\
\hline $\begin{array}{l}\text { Cymbopogon citratus } \\
\text { Stapf } \\
\text { (Poaceae) }\end{array}$ & citronnelle & $\begin{array}{l}\text { 1. Fièvre : Faire une infusion de feuilles et de racines } \\
\text { 2. Hypotension : Récolter des racines de citronnelle et } \\
\text { les faire bouillir jusqu'au changement de coloration de } \\
\text { l'eau. }\end{array}$ & $\begin{array}{l}\text { Feuilles et } \\
\text { racines }\end{array}$ & $\begin{array}{l}\text { 1. Boire } 1 \text { verre de cette } \\
\text { infusion matin, midi et soir } \\
\text { jusqu'à la guérison totale ; } \\
\text { 2. Boire } 1 \text { verre de cette } \\
\text { infusion matin, midi et soir } \\
\text { jusqu'à la guérison totale. }\end{array}$ & $\begin{array}{l}\text { citral, géranial, } \\
\text { myrcène }\end{array}$ \\
\hline $\begin{array}{l}\text { Dissotis rotundifolia } \\
\text { (Sm.) Triana. } \\
\text { (Melastomataceae) }\end{array}$ & $\begin{array}{l}\text { Ewuda } \\
\text { wanga }\end{array}$ & $\begin{array}{l}\text { Mal au nombril : Casser cinq noix de palme, les } \\
\text { écraser et les mélanger aux feuilles }\end{array}$ & Feuilles & Mâcher deux fois par jours & \\
\hline Emilia coccinea (Sims) & elo mvu & Mal d'oreille : Ecraser quelques feuilles fraîches & Feuilles & Mettre 2 gouttes à chaque & \\
\hline
\end{tabular}




\begin{tabular}{|c|c|c|c|c|c|}
\hline G. Don (Asteraceae) & (éwondo) & & & $\begin{array}{l}\text { oreille matin et soir jusqu'à } \\
\text { guérison complète }\end{array}$ & \\
\hline $\begin{array}{l}\text { Enantia chlorantha (Oliv.) } \\
\text { (Annonaceae) }\end{array}$ & Nfol & $\begin{array}{l}\text { Problèmes gastro-intestinaux : Tremper l'écorce dans } 1 \\
\text { litre d'eau, puis ajouter le citron et laisser refroidir. }\end{array}$ & Ecorce & $\begin{array}{l}\text { Boire } 1 \text { verre de ce } \\
\text { mélange matin et soir }\end{array}$ & $\begin{array}{l}\text { Berberine, palmatine } \\
\text { Jatrorrhizine, } \\
\text { columbamine, } \\
\text { pseudopalmatine } \\
\end{array}$ \\
\hline $\begin{array}{l}\text { Entandophragma } \\
\text { cylindricum (Sprague). } \\
\text { (Meliaceae) }\end{array}$ & Sapelli & $\begin{array}{l}\text { 1. Bronchite et Rhume : Faire une décoction d'écorce ; } \\
\text { 2. Furoncle et plaie : Récupérer les pulpes d'écorce. }\end{array}$ & Ecorce & $\begin{array}{l}\text { 1. Boire } 1 \text { verre de décocté } \\
\text { matin et soir ; } \\
\text { 2. Appliquer la pulpe sur le } \\
\text { furoncle ou la plaie }\end{array}$ & \\
\hline $\begin{array}{l}\text { Eremomastax speciosa } \\
\text { (Hochst.) Cufod. } \\
\text { (Acanthaceae) }\end{array}$ & $\begin{array}{l}\text { Dibokuboku } \\
\text { la busa di } \\
\text { mole }\end{array}$ & $\begin{array}{l}\text { 1. Erythème fessier : Ecraser les feuilles sèches et } \\
\text { mélanger la poudre avec de l'eau tiède et y ajouter de } \\
\text { l'huile de palme; } \\
\text { 2. Anémie: }\end{array}$ & Feuilles & $\begin{array}{l}\text { 1. Une purge deux fois par } \\
\text { semaine ; } \\
\text { 2. Boire } 1 / 2 \text { verre de ce } \\
\text { mélange deux fois par jour. }\end{array}$ & \\
\hline $\begin{array}{l}\text { Erythrophleum ivorense } \\
\text { A. Chev. } \\
\text { (Fabaceae) }\end{array}$ & $\begin{array}{l}\text { Lim, faux } \\
\text { tali, eloun } \\
\text { (beti) }\end{array}$ & $\begin{array}{l}\text { Variole : Extraire l'écorce et écraser avec les jeunes } \\
\text { rameaux }\end{array}$ & $\begin{array}{c}\text { Ecorces et } \\
\text { jeunes rameaux }\end{array}$ & $\begin{array}{l}\text { Frissonner la peau avec } \\
\text { l'eau dans laquelle on a } \\
\text { écrasé les écorces et } \\
\text { jeunes rameaux }\end{array}$ & $\begin{array}{c}\text { Cassaïne, } \\
\text { Cassaïdine ; } \\
\text { Erythrophéguine }\end{array}$ \\
\hline $\begin{array}{l}\text { Eucalyptus globulus } \\
\text { Labill. } \\
\text { (Myrtaceae) }\end{array}$ & Eucalyptus & $\begin{array}{l}\text { 1. Affections des voies respiratoires, anti } \\
\text { inflammatoire : Infuser } 20 \text { à } 30 \mathrm{~g} \text { de feuilles par litre } \\
\text { d'eau; } \\
\text { 2. Antiseptique: Infuser } 20 \text { à } 30 \mathrm{~g} \text { de feuilles par litre } \\
\text { d'eau. }\end{array}$ & Feuilles, bois & $\begin{array}{l}\text { 1. Boire } 3 \text { tasses de } \\
\text { l'infusion par jour avec du } \\
\text { miel ; } \\
\text { 2. Mettre sur l'endroit à } \\
\text { aseptiser } 4 \text { à } 10 \text { gouttes } \\
\text { reparties en journée. }\end{array}$ & Cinéol (ou eucalyptol) \\
\hline $\begin{array}{l}\text { Euphorbia hirta L. } \\
\text { (Euphorbiaceae) }\end{array}$ & rougette & $\begin{array}{l}\text { Hémorroïde et diarrhées : Ecraser les feuilles et } \\
\text { mélanger avec de l'huile et du sel dans } 1 \text { I d'eau }\end{array}$ & $\begin{array}{l}\text { Feuilles, plante } \\
\text { entière }\end{array}$ & $\begin{array}{l}\text { Boire } 1 \text { tasse de ce décocté } \\
\text { matin et soir }\end{array}$ & $\begin{array}{l}\text { choline, acide } \\
\text { shikimique, }\end{array}$ \\
\hline $\begin{array}{l}\text { Garcinia kola Heckel. } \\
\text { (Clusiaceae) }\end{array}$ & Bitter kola & Aphrodisiaque & Fruit & $\begin{array}{l}\text { Consommer un à deux } \\
\text { fruits par jour }\end{array}$ & $\begin{array}{l}\text { Caféine, } \\
\text { théobromine. }\end{array}$ \\
\hline $\begin{array}{l}\text { Hibiscus esculentus L. } \\
\text { (Malvaceae) }\end{array}$ & Gombo & $\begin{array}{l}\text { 1. Facilite l'accouchement, à prendre les dernières } \\
\text { semaines d'accouchement et contre les irritations de la } \\
\text { peau : Macérer les feuilles; } \\
\text { 2. Diurétique : Macérer les feuilles et mélanger dans } 1 \\
\text { litre d'eau. }\end{array}$ & $\begin{array}{l}\text { feuilles, racine, } \\
\text { graine }\end{array}$ & $\begin{array}{l}\text { 1. Frotter sur le ventre de la } \\
\text { femme enceinte pour } \\
\text { réduire les douleurs de } \\
\text { l'enfantement ou pour } \\
\text { éradiquer les irritations de } \\
\text { la peau; }\end{array}$ & Quinine \\
\hline
\end{tabular}




\begin{tabular}{|c|c|c|c|c|c|}
\hline & & & & $\begin{array}{l}\text { 2. Boire } 3 \text { verres de ce } \\
\text { mélange par jour pour } \\
\text { enfants, sans dose pour } \\
\text { adulte }\end{array}$ & \\
\hline $\begin{array}{l}\text { Hibiscus sabdarifa L. } \\
\text { (Malvaceae) }\end{array}$ & $\begin{array}{l}\text { Oseille de } \\
\text { guinée } \\
\text { (foléré) }\end{array}$ & $\begin{array}{l}\text { 1. Hémorroïdes : Faire une décoction de } 30 \mathrm{~g} \text { de fleurs } \\
\text { rouges par litre d'eau, laissé bouillir pendant } 10 \mathrm{mn} \text { et } \\
\text { filtrer; } \\
\text { 2. Diurétique : Mélanger les fleurs dans } 1 \text { I d'eau froide } \\
\text { ou chaude et laissé infuser pendant } 5 \mathrm{mn} \text {; } \\
\text { 3. Antiseptique : Macérer les fleurs pendant } 15 \mathrm{mn}\end{array}$ & Fleurs & $\begin{array}{l}\text { 1. Boire 1I de décoction } \\
\text { par jour ; } \\
\text { 2. Boire dans la journée ; } \\
\text { 3. Appliquer le produit de la } \\
\text { macération sur la blessure. }\end{array}$ & \\
\hline $\begin{array}{l}\text { Holarrhena floribunda } \\
\text { (G.Don.) Dur.\& Schinz } \\
\text { (Apocynaceae) }\end{array}$ & $\begin{array}{l}\text { Holarrhène } \\
\text { florifère }\end{array}$ & $\begin{array}{l}\text { 1. Vaginites et urétrites : Faire une décoction } \\
\text { d'écorces ; } \\
\text { 2. Blennorragie : Faire une décoction des feuilles ; } \\
\text { 3. Vers intestinaux : Faire une macération d'écorces. }\end{array}$ & Ecorces, feuilles & $\begin{array}{l}\text { 1. Boire } 1 \text { verre de décocté } \\
\text { matin et soir ; } \\
\text { 2. Boire } 1 \text { verre de décocté } \\
\text { matin et soir ; } \\
\text { 3. Boire } 1 \text { verre de la } \\
\text { macération matin et soir. }\end{array}$ & Conessine \\
\hline $\begin{array}{l}\text { Kalanchoe crenata } \\
\text { (Andrews) Haw. } \\
\text { (Crassulaceae) }\end{array}$ & Edibédibé & $\begin{array}{l}\text { Rhume (nourrisson), sinusite : Ramollir les feuilles à la } \\
\text { flamme, presser pour extraire le jus }\end{array}$ & Feuilles & $\begin{array}{l}\text { Mettre deux gouttes dans } \\
\text { les narines de l'enfant trois } \\
\text { fois par jour }\end{array}$ & \\
\hline $\begin{array}{l}\text { Mangifera indica Linn. } \\
\text { (Anacardiaceae) }\end{array}$ & Manguier & $\begin{array}{l}\text { 1. Rhumatisme : Faire une décoction d'écorces dans } 5 \\
\text { I d'eau; } \\
\text { 2. Mal de dents : Faire une décoction d'écorces dans } 1 \\
\text { I d'eau; } \\
\text { 3. Diarrhée et dysenterie amibienne : Faire une } \\
\text { décoction d'écorces et de feuilles dans } 1 \text { I d'eau ; } \\
\text { 4. Affections des voies urinaires : Faire une décoction } \\
\text { d'écorces dans } 1 \text { I d'eau ; } \\
\text { 5. Insuffisance rénale : Faire une décoction des racines } \\
\text { coupées en petits morceaux dans } 1 \text { I d'eau ; } \\
6 \text {. infection respiratoire : Faire une décoction d'écorces } \\
\text { dans } 1 \text { I d'eau. }\end{array}$ & $\begin{array}{l}\text { Ecorces, } \\
\text { Feuilles, racines }\end{array}$ & $\begin{array}{l}\text { 1. Tremper la serviette } \\
\text { dans la solution chaude et } \\
\text { masser ; } \\
\text { 2. Faire un bain de bouche } \\
\text { avec le décocté ; } \\
\text { 3. Boire } 1 \text { verre de décocté } \\
\text { matin et soir; } \\
\text { 4. Boire } 1 \text { verre de décocté } \\
\text { matin et soir; } \\
\text { 5. Boire } 1 \text { verre de décocté } \\
\text { matin et soir; } \\
6 . \text { Boire } 1 \text { verre de décocté } \\
\text { matin et soir. }\end{array}$ & $\begin{array}{l}\text { Choloine, Stachynine, } \\
\text { Mangiférine }\end{array}$ \\
\hline $\begin{array}{l}\text { Melissa officinalis L. } \\
\text { (Lamiaceae) }\end{array}$ & citronnelle & $\begin{array}{l}\text { Hypertension artérielle et jambes gonflées pour les } \\
\text { femmes enceintes : Bien laver les feuilles et faire une }\end{array}$ & Feuilles & $\begin{array}{l}\text { 1. Boire sans sucre, } 1 \text { tasse } \\
\text { matin, midi et soir }\end{array}$ & \\
\hline
\end{tabular}




\begin{tabular}{|c|c|c|c|c|c|}
\hline & & décoction dans 1I d'eau & & & \\
\hline $\begin{array}{l}\text { Myristica fragrans Houtt. } \\
\text { (Myristicaceae) }\end{array}$ & Muscadier & $\begin{array}{l}\text { Flatulences, fièvre : Faire une décoction des fruits dans } \\
1 \text { I d'eau; } \\
\text { 2. Ulcères gastriques : Verser une tasse d'eau } \\
\text { bouillante dans } 10 \mathrm{~g} \text { de noix de muscade en poudre ; } \\
\text { 3. Fièvre et saignement utérin abondant : Faire une } \\
\text { infusion des fruits dans } 1 \text { I d'eau ; }\end{array}$ & Fruit & $\begin{array}{l}\text { 1. Boire } 1 \text { verre de décocté } \\
\text { matin et soir ; } \\
\text { 2. Boire } 1 / 2 \text { tasse par jour ; } \\
\text { 3. Boire } 1 \text { verre de cette } \\
\text { infusion matin et soir ; }\end{array}$ & $\begin{array}{c}\text { Myristicine, } \\
\text { strychnine et brucine }\end{array}$ \\
\hline $\begin{array}{l}\text { Nicotiana tabaccum L. } \\
\text { (Solanaceae) }\end{array}$ & Tabac & $\begin{array}{l}\text { 1. Dépression : Faire une décoction des feuilles dans } 1 \\
\text { I d'eau; } \\
\text { 2. Vers intestinaux : Faire une macération des feuilles } \\
\text { dans } 1 \text { I d'eau; } \\
\text { 3. Gale : Bouillir } 5 \text { feuilles dans } 1 \text { I d'eau ; } \\
\text { 4. Traitement des poux : faire un décocté des feuilles } \\
\text { fraîches dans } 1 \text { I d'eau; } \\
\text { 5. Rhume : Ecraser les feuilles sèches ; } \\
\text { 6. Vermifuge : Macérer trois ou cinq feuilles fraîches ; }\end{array}$ & Feuilles & $\begin{array}{l}\text { 1. Boire } 1 \text { verre de décocté } \\
\text { matin et soir ; } \\
\text { 2. Boire } 1 \text { verre de macéré } \\
\text { matin et soir ; } \\
\text { 3. Embaumer tout le corps ; } \\
\text { 4. Se laver la tête } 2 \text { à } 3 \text { fois } \\
\text { par jour ; } \\
\text { 5. Appliquer la poudre dans } \\
\text { les narines ; } \\
\text { 6. Se purger avec le liquide } \\
\text { obtenu; } \\
\text { 7. Inhaler la poudre ; }\end{array}$ & $\begin{array}{l}\text { Nicotine, Atropine, } \\
\text { Curare, Muscarine, } \\
\text { Cotinine, Anatabine, } \\
\text { Nornicotine, } \\
\text { Anabasine }\end{array}$ \\
\hline $\begin{array}{l}\text { Ocimum basilicum L. } \\
\text { (Lamiaceae) }\end{array}$ & Basilic & $\begin{array}{l}\text { 1,2. Indigestion : Verser une } 1 / 2 \text { tasse d'eau bouillante } \\
\text { sur quatre cuillerées de feuilles écrasées et laissé } \\
\text { infuser dix minutes; } \\
\text { Toux des enfants }\end{array}$ & $\begin{array}{l}\text { Feuilles, fleurs, } \\
\text { partie entière }\end{array}$ & $\begin{array}{l}\text { 1. Boire } 1 / 2 \text { tasse d'infusion } \\
\text { toutes les quatre heures } \\
\text { pendant la journée. } \\
2 \text {. Boire } 1 \text { cuillerée à soupe } \\
\text { toutes les huit heures aux } \\
\text { enfants comme remède } \\
\text { contre la toux }\end{array}$ & $\begin{array}{l}\text { Linalol, Eugenol, } \\
\text { Estragol }\end{array}$ \\
\hline $\begin{array}{l}\text { Panax ginseng C.A. Mey. } \\
\text { (Areliaceae) }\end{array}$ & Ginseng & $\begin{array}{l}\text { Dynamisation des défenses immunitaires, } \\
\text { Ralentissement du vieillissement, prévention des } \\
\text { maladies dégénératives, normalisation des } \\
\text { dysfonctionnements endocriniens, accélération des } \\
\text { processus de régénération : Faire une infusion des } \\
\text { racines dans } 1 \text { I d'eau }\end{array}$ & Racines & $\begin{array}{l}\text { Boire } 1 \text { verre de l'infusion } \\
\text { matin, midi et soir }\end{array}$ & ginsénosides \\
\hline Panax quinquerfolium & Ginseng & Fatigue, stress, faiblesse sexuelle, diabète : Faire une & Racines & Boire 1 verre de l'infusion & \\
\hline
\end{tabular}




\begin{tabular}{|c|c|c|c|c|c|}
\hline $\begin{array}{l}\text { Herbs \& Health. } \\
\text { (Areliaceae) }\end{array}$ & Americain & infusion des racines dans 1 I d'eau & & matin, midi et soir & \\
\hline $\begin{array}{l}\text { Passiflora incarnata L. } \\
\text { (Passifloraceae) }\end{array}$ & Passiflore & $\begin{array}{l}\text { Insomnies, fatigue, maux de tête d'origine nerveuse, } \\
\text { tranquillisant : Faire une macération de la plante } \\
\text { entière }\end{array}$ & Toute la plante & $\begin{array}{l}15 \text { à } 20 \text { goutes dans } 1 / 2 \\
\text { tasse d'eau ( } 3 \text { à } 10 \text { gouttes } \\
\text { pour enfants) après chaque } \\
\text { heure }\end{array}$ & Harmine, Harmol, \\
\hline $\begin{array}{l}\text { Pausinystalia yohimbe } \\
\text { (K. Schum.) Pierre ex } \\
\text { Beille. } \\
\text { (Rubiaceae) }\end{array}$ & $\begin{array}{c}\text { Yohimbe } \\
\text { (man power) }\end{array}$ & $\begin{array}{l}\text { Aphrodisiaque, mal de ventre, frigidité, impuissance: } \\
\text { Gratter l'écorce et mélanger la poudre obtenue dans du } \\
\text { whisky ou dans du coca cola et laisser macérer deux } \\
\text { jours }\end{array}$ & Ecorces & $\begin{array}{l}\text { Boire deux bouchons tous } \\
\text { les deux jours pendant un } \\
\text { mois et recommencer le } \\
\text { traitement tous les six mois } \\
\text { (aphrodisiaque, frigidité et } \\
\text { impuissance) ;. } \\
\text { Boire jusqu'à la guérison du } \\
\text { mal de ventre. }\end{array}$ & Yohimbine \\
\hline $\begin{array}{l}\text { Persea americana } \\
\text { Mill. (Lauraceae) }\end{array}$ & Piya & $\begin{array}{l}\text { puissant aphrodisiaque, dysenterie : Prendre } 6 \text { à } 8 \\
\text { bourgeons, verser dessus } 1 \text { I d'eau bouillante, couvrir } \\
\text { et laisser infuser } 15 \mathrm{mn} \text {, puis filtrer }\end{array}$ & fruits, fleurs & $\begin{array}{l}\text { Boire } 1 \text { tasse matin, midi et } \\
\text { soir avant les repas ; }\end{array}$ & \\
\hline $\begin{array}{l}\text { Petroselinum sativum } \\
\text { (Mill.) Nyman ex A. W. } \\
\text { Hill. (Apiaceae) }\end{array}$ & persil & $\begin{array}{l}\text { 1. Jaunisse et hypertension artérielle : Faire une } \\
\text { décoction de toute la plante dans } 1 \text { I d'eau ; } \\
\text { 2. Règles douloureuses : Faire une infusion d'une main } \\
\text { de persil dans } 1 \text { I d'eau bouillante pendant } 10 \mathrm{mn} \text {; } \\
\text { 3. Rétention urinaire : Décoction de } 3 \text { à } 5 \text { feuilles dans } \\
1 / 2 \text { litre d'eau et laissé reposer } 10 \text { à } 15 \mathrm{mn} \text {. }\end{array}$ & Toute la plante & $\begin{array}{l}\text { 1. Boire } 1 \text { verre de décocté } \\
\text { matin et soir ; } \\
\text { 2. Boire au cours de la } \\
\text { journée ; } \\
\text { 3. Boire } 1 / 2 \text { tasse trois fois } \\
\text { par jour. }\end{array}$ & $\begin{array}{l}\text { Apiol, muristicine, } \\
\text { bergaptène, huile } \\
\text { avec l'acide } \\
\text { pétrosélinique }\end{array}$ \\
\hline $\begin{array}{l}\text { Phyllathus amarus } \\
\text { Schumach. \& Thonn., } \\
\text { Kongel. Danske Vidensk. } \\
\text { Selsk. Skr. } \\
\text { (Euphorbiaceae) }\end{array}$ & $\begin{array}{l}\text { herbe au } \\
\text { chagrin }\end{array}$ & $\begin{array}{l}\text { Ulcère gastrique, paludisme, diarrhée, dysenterie: } \\
\text { Faire une décoction de feuilles dans } 1 \text { I d'eau }\end{array}$ & Feuilles & $\begin{array}{l}\text { Boire } 1 \text { verre décocté matin } \\
\text { et soir }\end{array}$ & $\begin{array}{l}\text { Quinolizidine, } \\
\text { phyllanthine, } \\
\text { sécurinine, } \\
\text { norsécurinine, } \\
\text { isobubbialine, } \\
\text { épibubbialie } \\
\end{array}$ \\
\hline $\begin{array}{l}\text { Psidium guajava Linn. } \\
\text { (Myrtaceae) }\end{array}$ & Goyavier & $\begin{array}{l}\text { 1. Diarrhées ; } \\
\text { 2. Dysenterie amibienne : Bouillir les feuilles dans } 1 \text { I } \\
\text { d'eau avec un peu de sel et de sucre. }\end{array}$ & Feuilles & $\begin{array}{l}\text { 1. Mâcher et avaler les } \\
\text { jeunes feuilles ; } \\
\text { 2. Boire } 1 \text { verre matin et } \\
\text { soir. }\end{array}$ & $\begin{array}{l}\text { Guajaverine, Bis } \\
\text { indolite, quinoniques }\end{array}$ \\
\hline Rauvolfia vomitoria Afzel. & Baie de & 1. Rhumatisme et affections pulmonaires : Faire une & Feuilles, racines & 1. Boire 1 verre de décocté & Quinine,alcaloïdes \\
\hline
\end{tabular}




\begin{tabular}{|c|c|c|c|c|c|}
\hline (Apocynaceae) & l'aubépine & $\begin{array}{l}\text { décoction des feuilles dans } 1 \text { I d'eau; } \\
\text { 2. Vers intestinaux : Faire une décoction des racines et } \\
\text { des écorces; } \\
\text { 3. Affections pulmonaires : Faire une décoction des } \\
\text { feuilles dans } 10 \text { I d'eau. } \\
\text { 4. facilite l'accouchement : Faire une décoction des } \\
\text { feuilles et des racines. } \\
\text { 5. Epilepsies : Faire une décoction des racines }\end{array}$ & et écorces & $\begin{array}{l}\text { matin et soir ; } \\
\text { 2. Boire } 1 \text { verre de décocté } \\
\text { matin et soir ; } \\
\text { 3. Se baigner. }\end{array}$ & $\begin{array}{l}\text { indoliques, } \\
\text { punarnavine, } \\
\text { agroclavine }\end{array}$ \\
\hline $\begin{array}{l}\text { Senna alata (L.) Roxb } \\
\text { (Fabaceae) }\end{array}$ & Dartrier & $\begin{array}{l}\text { 1. Dartre : Piler les feuilles jusqu'à l'obtention d'une } \\
\text { pâte ; } \\
\text { 2. Constipation : Faire bouillir les feuilles ; } \\
\text { 3. Jaunisse : Faire bouillir les feuilles ; } \\
\text { 4. Teigne : Ecraser les feuilles et ajouter le jus de } \\
\text { citron; } \\
\text { 5. Eczéma, herpès : Broyer les feuilles ; } \\
\text { 6. Infection des plaies : Broyer légèrement les feuilles ; } \\
\text { 7. Douleurs rhumatismales : Macération dans l'eau, et } \\
\text { extraction du jus. }\end{array}$ & Feuilles & $\begin{array}{l}\text { 1. Frotter la pâte sur le } \\
\text { corps; } \\
\text { 2. Boire } 1 \text { verre de } \\
\text { décocté : } \\
\text { 3. Ajouter le sel gemme } \\
\text { dans le décocté et boire ; } \\
\text { 4. Appliquer la pâte sur les } \\
\text { parties malades ; } \\
\text { 5. Appliquer sous forme de } \\
\text { cataplasme ; } \\
\text { 6. Déposer le broyat sur les } \\
\text { parties infectées; } \\
\text { 7. La dose administrée } \\
\text { dépend de du } \\
\text { degré d'infections. }\end{array}$ & $\begin{array}{l}\text { Anthracenosides, } \\
\text { tyramine }\end{array}$ \\
\hline $\begin{array}{l}\text { Solanum lycopersicum } \\
\text { Mill. } \\
\text { (Solanaceae) }\end{array}$ & Tomate & $\begin{array}{l}\text { Maladies cardiovasculaires, anti-oxydant, anti- } \\
\text { cancéreux, hémorroïdes, inflammation, piqures } \\
\text { d'insectes, constipation, diabète, cardiaque, obésité, } \\
\text { anémie }\end{array}$ & $\begin{array}{l}\text { Feuilles tiges } \\
\text { fruits, fibres, } \\
\text { graines, pulpe }\end{array}$ & & $\begin{array}{l}\text { Lycopène, Gluco- } \\
\text { alcaloides (tomatine), } \\
\text { hétéroside, } \\
\text { tomatidine }\end{array}$ \\
\hline $\begin{array}{l}\text { Solanum melongena } \\
\text { Linn. (Solanaceae) }\end{array}$ & aubergine & $\begin{array}{l}\text { diabète, hypertension, rhumatisme, constipation, } \\
\text { cholestérol, coliques, sédatif, coliques }\end{array}$ & $\begin{array}{l}\text { Feuilles, fruits, } \\
\text { racine }\end{array}$ & & Nicotine \\
\hline $\begin{array}{l}\text { Solanum nigrum Linn. } \\
\text { (Solanaceae) }\end{array}$ & morelle noire & Infections cutanée, douleur abdominale & $\begin{array}{l}\text { Baies, feuilles, } \\
\text { racine }\end{array}$ & & salonine \\
\hline $\begin{array}{l}\text { Solanum tuberosum } \\
\text { Linn. } \\
\text { (Solanaceae) }\end{array}$ & $\begin{array}{l}\text { pomme de } \\
\text { terre }\end{array}$ & $\begin{array}{l}\text { Brûlures légères : Peler les pommes, les laver, les } \\
\text { râper à l'aide d'un grattoir }\end{array}$ & Tubercules & Appliquer sur la lésions & $\begin{array}{l}\text { Solamine, Pectine, } \\
\text { Albumine, histamine, } \\
\text { tyramine, } \\
\text { méthylamine, choline, }\end{array}$ \\
\hline
\end{tabular}




\begin{tabular}{|c|c|c|c|c|c|}
\hline & & & & & , chaconine \\
\hline $\begin{array}{l}\text { Theobroma cacao } \\
\text { Linn. } \\
\text { (Malvaceae) }\end{array}$ & Cacaoyer & $\begin{array}{l}\text { 1. Diurétique : Sécher et écraser les graines jusqu'à } \\
\text { l'obtention de la poudre et en faire une infusion; } \\
\text { 2. Diurétique : Faire une décoction des graines de } \\
\text { cacao dans } 1 \text { I d'eau ; } \\
\text { Douleurs musculaires : Faire une décoction de } 50 \mathrm{~g} \text { de } \\
\text { l'écorce dans } 1 \text { I d'eau. }\end{array}$ & $\begin{array}{l}\text { Graines, } \\
\text { écorces }\end{array}$ & $\begin{array}{l}\text { 1. Boire } 1 \text { verre de l'infusion } \\
\text { matin et soir ; } \\
\text { 2. Boire } 3 \text { tasses de } \\
\text { décocté par jour ; } \\
\text { 3. Masser les douleurs } \\
\text { musculaires sur les zones } \\
\text { affectées } 6 \text { à } 8 \text { fois par jour. }\end{array}$ & $\begin{array}{l}\text { caféine, théobromine, } \\
\text { tanins théophylline, } \\
\text { tyramine, xanthines, } \\
\text { phényléthanamine, } \\
\text { Endorphines. }\end{array}$ \\
\hline $\begin{array}{l}\text { Thymus vulgaris Linn. } \\
\text { (Lamiaceae) }\end{array}$ & Thym & $\begin{array}{l}\text { Vomissement : Faire une infusion des feuilles dans } 11 \\
\text { d'eau }\end{array}$ & Feuilles & $\begin{array}{l}\text { Boire } 1 \text { verre de décocté } \\
\text { matin et soir }\end{array}$ & \\
\hline $\begin{array}{l}\text { Tinospora bakis (A.Rich) } \\
\text { Miers. } \\
\text { (Menispermaceae) }\end{array}$ & $\begin{array}{l}\text { bakis bakis } \\
\text { (wolof) }\end{array}$ & Fièvre : Faire une décoction des racines dans 1 I d'eau & Racines & $\begin{array}{l}\text { Boire } 1 \text { verre de décocté } \\
\text { matin et soir }\end{array}$ & colombine, palmatine \\
\hline $\begin{array}{l}\text { Voacanga africana Stapf } \\
\text { (Apocynaceae) }\end{array}$ & & & $\begin{array}{c}\text { Graines, } \\
\text { écorces, } \\
\text { feuilles, racine }\end{array}$ & & $\begin{array}{l}\text { Voacamine, } \\
\text { Tabersonine }\end{array}$ \\
\hline $\begin{array}{l}\text { Voacanga thouarsii } \\
\text { Roem. \& Schult. } \\
\text { (Apocynaceae) }\end{array}$ & Voacanga & $\begin{array}{l}\text { 1. Poux : Préparer une poudre à partir des écorces } \\
\text { écrasées ; } \\
\text { Ulcères : Faire une infusion des graines. }\end{array}$ & Graines, écorce & $\begin{array}{l}\text { 1. Appliquer la poudre sur } \\
\text { la tête ; } \\
\text { 2. Boire matin et soir avant } \\
\text { le repas. }\end{array}$ & $\begin{array}{l}\text { Tabersonine, } \\
\text { voacamine }\end{array}$ \\
\hline $\begin{array}{l}\text { Zea mays Linn. } \\
\text { (Poaceae) }\end{array}$ & maïs & $\begin{array}{l}\text { Amaigrissant : Faire une décoction pendant } 1 \mathrm{~h} \text { des } \\
\text { barbes fraîches ou sèches }\end{array}$ & $\begin{array}{c}\text { Stigmates } \\
\text { (barbe de maïs) }\end{array}$ & prendre $500 \mathrm{ml}$ par jour & $\begin{array}{l}\text { Albumine, Glucose, } \\
\text { Amino-acide }\end{array}$ \\
\hline $\begin{array}{l}\text { Zingiber officinale Rosc. } \\
\text { (Zingiberaceae) }\end{array}$ & gingembre & $\begin{array}{l}\text { Toux, fatigue nerveuse, indigestion : Faire une } \\
\text { macération des racines }\end{array}$ & Racines & $\begin{array}{l}\text { Boire } 1 \text { cuillère de macéré } \\
\text { matin, midi et soir }\end{array}$ & $\begin{array}{l}\text { Capsaïcine, pipérine, } \\
\text { curcumine }\end{array}$ \\
\hline
\end{tabular}

